\title{
Linfografía como opción diagnóstica y terapéutica en la fuga quilosa posoperatoria de cáncer colorrectal
}

\author{
Lymphography as a diagnostic and therapeutic option in postoperative chylous leakage \\ of colorectal cancer
}

\author{
Aida Suárez-Sánchez*, María Fernández-Hevia, Tamara Díaz-Vico, Manuel García-Munar, \\ Carmen García-Gutiérrez, Daniel Fernández-Martínez, Jorge L. Otero-Diez, \\ Luis J. García-Flórez y José E. Granero-Trancón \\ Sección de Coloproctología, Servicio de Cirugía General, Hospital Universitario Central de Asturias, Oviedo, España
}

\begin{abstract}
Resumen
La fuga quilosa es una complicación muy poco frecuente tras la cirugía colorrectal. Se presenta el caso de un paciente de 70 años con neoplasia de recto medio intervenido de forma electiva tras un ciclo largo de neoadyuvancia mediante una resección anterior de recto por laparoscopia. El cuarto día de posoperatorio presentó un drenaje pélvico de aspecto quiloso y el día 13 se confirmó la fuga quilosa en la linfografía. Posteriormente el débito se redujo de forma rápida. La linfografía no solo es un método diagnóstico, sino que en el 35-70\% de los casos puede también ser terapéutica.
\end{abstract}

Palabras clave: Ascitis quilosa. Cáncer colorrectal. Fístula quilosa. Linfografía.

\begin{abstract}
Chylous leakage is an extremely rare complication after colorectal surgery. We report the case of a 70 year-old male with a mid-rectal cancer who underwent a laparoscopic anterior resection of the rectum after long course neoadjuvant therapy. On postoperative day 4 the patient presented with chylous pelvic drainage, and a chylous leakage was proved by lymphography on postoperative day 13. Hereinafter, the drainage was drastically reduced. The lymphography is not only a diagnostic technique, but it can be also a therapeutic method in up to $35-70 \%$ of the cases.
\end{abstract}

Key words: Chylous ascites. Colorectal cancer. Chylous leakage. Lymphography.

\section{Introducción}

La fuga quilosa o ascitis quilosa se define como la extravasación peritoneal de líquido lechoso rico en triglicéridos ${ }^{1,2}$. Aunque es rara, la causa más frecuente en adultos es la patología maligna. Otras causas descritas son traumatismos abdominales, pancreatitis, radioterapia o secundaria a cirugía abdominal ${ }^{3}$. La incidencia tras esta última es del 0,3-11\%, en relación con amplias linfadenectomías retroperitoneales o con cirugía aórtica ${ }^{4,5}$. En cirugía colorrectal es una complicación muy poco frecuente, lo que puede retrasar su diagnóstico y su tratamiento, con prolongación de 
la estancia postoperatoria y, en el caso de las enfermedades oncológicas, retraso en el inicio del tratamiento adyuvante ${ }^{6}$. Su incidencia es del 1-6\%, y es más frecuente en casos de linfadenectomías extendidas. El objetivo de este trabajo es la presentación de un caso clínico que hemos tenido la oportunidad de atender en nuestro centro.

\section{Caso clínico}

Varón de 70 años con diagnóstico de neoplasia de recto medio cT3N1M0 (estadio IIIb) que recibió tratamiento con radioquimioterapia y fue intervenido en la novena semana después de la finalización de la neoadyuvancia. Se realizó una resección anterior baja de recto por laparoscopia con ileostomía de protección. Se reinició la tolerancia oral el primer día del posoperatorio. En el cuarto día se evidenció la salida de líquido blanquecino por el drenaje pélvico (débito de $400 \mathrm{~cm}^{3}$ al día), sin sintomatología abdominal asociada ni alteraciones analíticas (Fig. 1). La tomografía computarizada (TC) abdominal con enema de Gastrografin ${ }^{\circledR}$ descartó la fuga anastomótica y la presencia de colecciones intraabdominales. Ante la sospecha de fuga quilosa se analizó el líquido del drenaje, que mostró unos valores de triglicéridos de $900 \mathrm{mg} / \mathrm{dl}$, compatible con la sospecha diagnóstica. Se inició tratamiento conservador dado el buen estado clínico del paciente, con dieta baja en grasas y antibioticoterapia empírica, manteniéndose asintomático. Debido al alto débito del drenaje de forma mantenida, se decidió realizar una linfografía estándar con uso de esferas de lipiodol el día 13 de posoperatorio. Para ello se realizó una punción guiada por ecografía de ganglios linfáticos en ambas regiones inguinales y se confirmó la extravasación de contraste paravertebral izquierda proyectada en la vecindad de los clips de la sección de la arteria mesentérica inferior (Fig. 2). Tras la prueba, el débito disminuyó de manera importante, pudiendo retirarse el drenaje 3 días después y dar el alta el día 17. En controles posteriores el paciente no presenta signos de recidiva de su complicación ni de su enfermedad.

\section{Discusión}

La fuga quilosa es una complicación poco habitual que se produce por la lesión inadvertida de los vasos linfáticos durante el procedimiento quirúrgico. No existe mucha literatura al respecto y posiblemente su incidencia esté subestimada. Tras la cirugía colorrectal

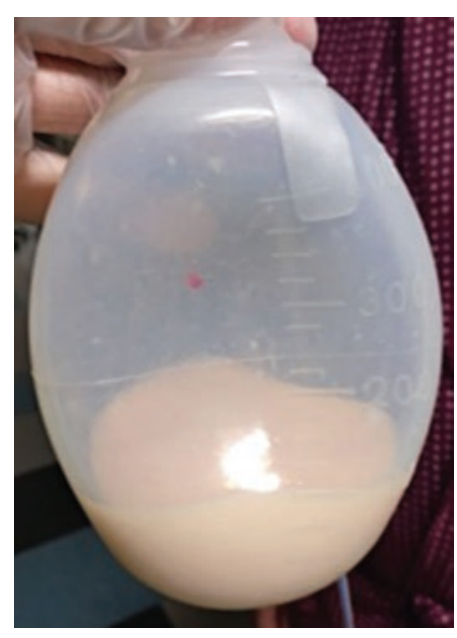

Figura 1. Drenaje quiloso.

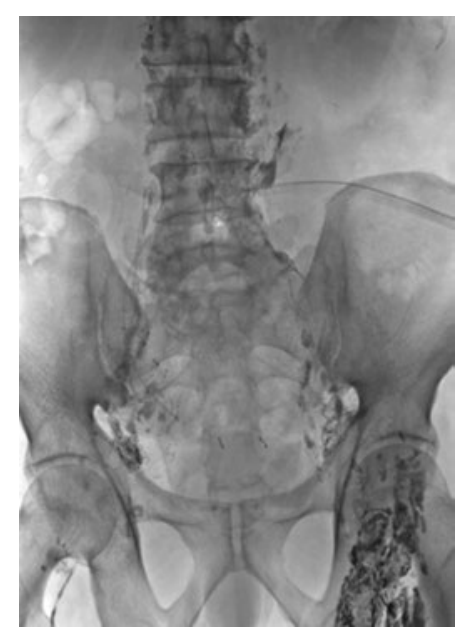

Figura 2. Linfografía. Fuga adyacente a clips de arteria mesentérica inferior.

se ha descrito hasta en el $6.6 \%$ de los pacientes, más frecuentemente tras colectomías derechas y en linfadenectomías extendidas (D3) ${ }^{7-9}$. Varios estudios han descrito factores de riesgo para la aparición de ascitis quilosa posoperatoria, como la edad, los niveles de albúmina, el número de ganglios linfáticos resecados, la pérdida hemática intraoperatoria y la realización de amplias linfadenectomías ${ }^{4,7,9-13}$.

La clínica más frecuente es la distensión abdominal no dolorosa, aunque en la mayoría de los casos se trata de fugas quilosas dirigidas con drenajes abdominales, por lo que son asintomáticas. El retraso en el diagnóstico y el tratamiento es habitual, pudiendo aparecer anorexia, debilidad, desnutrición proteica e incluso la muerte. Es muy infrecuente que se presente 
en forma de abdomen agudo, en cuyo caso se definiría como peritonitis quilosa aguda ${ }^{10}$. En las fugas quilosas no drenadas, será necesario realizar una paracentesis diagnóstica o un drenaje percutáneo para completar el diagnóstico. Hay que tener en cuenta que las paracentesis de repetición aumentan el riesgo de infección, por lo que no se debe abusar de este procedimiento $0^{7,10}$.

El diagnóstico suele realizarse a partir del cuarto día de posoperatorio, como en nuestro caso, coincidiendo con la progresión dietética. El color lechoso del drenaje es la primera sospecha diagnóstica. Otras pruebas complementarias son el análisis bioquímico del líquido y las pruebas de imagen, como la ecografía abdominal o la $\mathrm{TC}^{7,9,10}$.

El tratamiento conservador es el más utilizado y resulta eficaz en la mayoría de los casos. Consiste en dieta absoluta con nutrición parenteral y somatostatina u octreotida ${ }^{7-10,14}$, o dieta con triglicéridos de cadena media. La linfografía o la linfogammagrafía se utilizaban fundamentalmente para la localización del punto de fuga, pero se ha visto que tienen utilidad terapéutica. Desde el trabajo inicial de Cope en 1998 para el tratamiento del quilotórax, se ha publicado mucha literatura al respecto sobre la embolización linfática como tratamiento de este ${ }^{15,16}$. Sin embargo, existe poca experiencia sobre la ascitis quilosa y la linfografía.

Existen varios tipos de linfografía en función de su complejidad; las más sencillas y más utilizadas como técnicas diagnósticas son la que utiliza esferas de lipiodol, la asociada a embolización y la BORALE (Endolymphatic Balloon-Occluded Retrograde Abdominal Lymphangiography Embolization). La linfografía sin embolización no solo permite identificar el punto de fuga, sino que también ayuda a cerrarlo, con éxito hasta en el $70 \%$ de los casos. La linfografía con embolización con coils, pegamento u otros agentes esclerosantes presenta un éxito de hasta el $88 \%$. La técnica más avanzada, denominada BORALE, puede ser una alternativa en aquellos casos en los que con las variedades anteriores no se llegue a identificar la fuga. La evolución de la linfografía permite con éxito el diagnóstico y el tratamiento en la mayoría de las fístulas posoperatorias, con un riesgo mínimo para los pacientes ${ }^{17-19}$.

La necesidad de intervención quirúrgica es muy infrecuente, llevándose a cabo solo en casos refractarios al tratamiento conservador. Teniendo en cuenta que los pacientes con fuga quilosa refractaria pueden presentar un estado de malnutrición importante, es recomendable la optimización previa a la cirugía, así como la utilización de contrastes o una ingesta grasa la noche anterior a la intervención para maximizar las posibilidades de localización de la fístula. Las opciones quirúrgicas como el shunt peritoneovenoso, el cierre de la fístula o la resección intestinal no están exentas de complicaciones. Por ello, en casos de alto riesgo quirúrgico con fallo del tratamiento conservador se pueden plantear alternativas paliativas (shunts intrahepáticos portosistémicos [TIPS] ${ }^{10}$.

Existe poca literatura en relación con las consecuencias a largo plazo, y hay discrepancias al respecto. Algunos grupos señalan un peor pronóstico en estos pacientes ${ }^{14}$; en cambio, otros no lo asocian, aunque puntualizan que la inmunosupresión que conlleva la ascitis quilosa puede afectar a la supervivencia?.

\section{Conclusiones}

La fuga quilosa es una complicación muy rara en la cirugía colorrectal. Su diagnóstico temprano y su manejo adecuado permiten una alta tasa de resolución. Aunque el tratamiento conservador es eficaz en la mayoría de los casos, la linfografía o la linfogammagrafía pueden ser herramientas tanto diagnósticas como terapéuticas. La evolución de las técnicas radiológicas mínimamente invasivas permite resolver un alto porcentaje de casos de forma segura y eficaz, disminuyendo la necesidad de rescate quirúrgico. La cirugía se reserva para los casos refractarios al tratamiento conservador y no está exenta de complicaciones ${ }^{6}$.

\section{Financiamiento}

Los autores declaran que no ha habido ningún tipo de subvención ni financiamiento para la realización de este artículo.

\section{Conflicto de intereses}

Los autores declaran que ninguno está sujeto a ningún conflicto de intereses de ningún tipo.

\section{Responsabilidades éticas}

Protección de personas y animales. Los autores declaran que para esta investigación no se han realizado experimentos en seres humanos ni en animales.

Confidencialidad de los datos. Los autores declaran que han seguido los protocolos de su centro de trabajo sobre la publicación de datos de pacientes. 
Derecho a la privacidad y consentimiento informado. Los autores han obtenido el consentimiento informado de los pacientes y/o sujetos referidos en el artículo. Este documento obra en poder del autor de correspondencia.

\section{Bibliografía}

1. Gómez-Martín JM, Martínez-Molina E, Sanjuanbenito A, Martín-Illana E, Arrieta F, Balsa JA, et al. Chylous ascites secondary to acute pancreatitis: a case report and review of literature. Nutr Hosp. 2012;27: 314-8.

2. Chan KY, Teoh CM, Sukumar N. Chylous ascites after anterior resection for rectal carcinoma: a rare but significant incident. Asian J Surg. 2006;29:46-8

3. Park KT, Adikibi B, MacKinlay GA, Gillett PM, Sylvester KG, Kerner JA. Chylous ascites after laparoscopic Nissen fundoplication. Dig Dis Sci. 2012;57:28-31.

4. Assumpcao L, Cameron JL, Wolfgang CL, Edil B, Choti MA, Herman JM, et al. Incidence and management of chyle leaks following pancreatic resection: a high volume single-center institutional experience. J Gastrointest Surg. 2008;12:1915-23.

5. Weniger M, D'Haese JG, Angele MK, Kleespies A, Werner J, Hartwig W. Treatment options for chylous ascites after major abdominal surgery: a systematic review. Am J Surg. 2016;211:206-13.

6. Nadolski GJ, Chauhan NR, Itkin M. Lymphangiography and lymphatic embolization for the treatment of refractory chylous ascites. Cardiovasc Intervent Radiol. 2018;41:415-23.

7. Baek SJ, Kim SH, Kwak JM, Kim J. Incidence and risk factors of chylous ascites after colorectal cancer surgery. Am J Surg. 2013;206: 555-9.
8. Nishigori H, Ito M, Nishizawa $\mathrm{Y}$, Koyama A, Koda T, Nakajima K, et al. Postoperative chylous ascites after colorectal cancer surgery. Surg Today. 2012;42:724-8.

9. Lee SY, Kim CH, Kim YJ, Kim HR. Chylous ascites after colorectal cancer surgery: risk factors and impact on short-term and long-term outcomes. Langenbecks Arch Surg. 2016;401:1171-7.

10. Aalami $\mathrm{OO}$, Allen $\mathrm{DB}$, Organ $\mathrm{CH}$. Chylous ascites: a collective review. Surgery. 2000;128:761-78.

11. Yilmaz M, Akbulut S, Isik B, Ara C, Ozdemir F, Aydin C, et al. Chylous ascites after liver transplantation: incidence and risk factors. Liver Transpl. 2012;18:1046-52

12. Tulunay G, Ureyen I, Turan T, Karalok A, Kavak D, Ozgul N, et al. Chylous ascites: analysis of 24 patients. Gynecol Oncol. 2012;127:191-7.

13. Evans JG, Spiess PE, Kamat AM, Wood CG, Hernandez M, Pettaway $\mathrm{CA}$, et al. Chylous ascites after post-chemotherapy retroperitoneal lymph node dissection: review of the M. D. Anderson experience. J Urol. 2006;176:1463-7.

14. Matsuda T, Fujita H, Kunimoto $Y$, Kimura T, Ogino K. Chylous ascites as a complication of laparoscopic colorectal surgery. Asian $\mathrm{J}$ Endosc Surg. 2013;6:279-84.

15. Cope C. Diagnosis and treatment of postoperative chyle leakage via percutaneous transabdominal catheterization of the cisterna chyli: a preliminary study. J Vasc Interv Radiol. 1998;9:727-34.

16. Cope C, Kaiser LR. Management of unremitting chylothorax by percutaneous embolization and blockage of retroperitoneal lymphatic vessels in 42 patients. J Vasc Interv Radiol. 2002;13:1139-48.

17. Majdalany BS, El-Haddad G. Contemporary lymphatic interventions for post-operative lymphatic leaks. Transl Androl Urol. 2020;9(S1):S104-13.

18. Majdalany BS, Khayat M, Downing T, Killoran TP, El-Haddad G, Khaja MS, et al. Lymphatic interventions for isolated, iatrogenic chylous ascites: a multi-institution experience. Eur J Radiol. 2018;109:41-7.

19. Srinivasa RN, Gemmete JJ, Osher ML, Hage AN, Chick JFB. Endolymphatic Balloon-Occluded Retrograde Abdominal Lymphangiography (BO$\mathrm{RAL}$ ) and Embolization (BORALE) for the diagnosis and treatment of chylous ascites: approach, technical success, and clinical outcomes. Ann Vasc Surg. 2018;49:49-56. 